\title{
Histopathology and serial, multimodal magnetic resonance imaging in a multiple sclerosis variant
}

\author{
S Lindquist ${ }^{1,2}, N$ Bodammer $^{1}$, J Kaufmann ${ }^{1}, F$ König ${ }^{3}$, H-J Heinze $e^{1,2}$, W Brück ${ }^{3}$ and \\ M Sailer ${ }^{1}$
}

\begin{abstract}
Defining tools in magnetic resonance imaging (MRI) representing specific pathological processes is needed to understand the complex relationship between inflammation, myelin breakdown, axonal injury and clinical symptoms in multiple sclerosis (MS) and its variants. Here, we describe a case of histologically-defined MS, in which the radiological appearance of the lesion and clinical course support the diagnosis of Balo's concentric sclerosis. Serial magnetization transfer, diffusion tensor imaging and ${ }^{1} \mathrm{H}$-magnetic resonance spectroscopy, from 14 days to 13 months after biopsy, allow the contextual interpretation of specific pathological changes. In our case, acute inflammation was sensitively traced by fractional anisotropy and increased lactate in spectroscopy. In contrast, magnetization transfer ratio and the apparent diffusion coefficient monitor the sequential loss of tissue in selected rings of the lesion. The delay from the peak of symptoms in a dramatic clinical course to the maximum tissue destruction indicated through MRI suggests that compromise of axonal function may be decisive for the acute clinical situation. This is the first report comparing ${ }^{1} \mathrm{H}-$ magnetic resonance spectroscopy, magnetization transfer and diffusion tensor imaging with histopathology in a patient with Balo's concentric sclerosis. Multiple Sclerosis 2007; 13: 471-482. http://msj.sagepub.com
\end{abstract}

Key words: Balo's concentric sclerosis; diffusion-weighted imaging; histology; magnetic resonance imaging; magnetization transfer; multiple sclerosis

\section{Introduction}

Balo's concentric sclerosis, a specific variant of multiple sclerosis (MS), is a rather acute, often monophasic and rapidly progressive, inflammatory demyelinating disease mainly affecting young adults. A single or very few lesions, often large and not readily distinguishable from neoplasms, show alternating bands of demyelination, and relatively intact white matter, resulting from preservation of tissue rather than remyelination $[1,2]$. Hypoxic preconditioning of oligodendrocytes is now thought to be a major factor contributing to the pathognomonic onion-bulb appearance of the lesions [3].

The pathomechanisms in MS and its variants are poorly understood [4]. Histopathological studies in humans, although highly valuable, are limited by the lack of longitudinal analysis. Lesions can be detected non-invasively via magnetic resonance imaging (MRI), however, conventional techniques lack pathological specificity. In a study of six biopsied cases neither $T_{1^{-}}$nor $T_{2}$-intensities nor gadolinium (Gd)-enhancement were demonstrated as specific to either age of the lesion or a predominant pathological process [5]. Modern MRI techniques, such as magnetization transfer imaging (MTI) and diffusion tensor imaging (DTI), promise to better differentiate the underlying pathology. Correlating well with disability in patients with MS, a decreased magnetization transfer ratio (MTR) is considered a measure for tissue destruction [6]. Although not specific for demyelination, as MTR also correlates with axonal density [7] and decreases

\footnotetext{
${ }^{1}$ Department of Neurology II, Otto-von-Guericke University, 39120 Magdeburg, Germany

2 Leibniz Institute for Neurobiology, 39118 Magdeburg, Germany

3 Department of Neuropathology, University of Goettingen, Goettingen, Germany

Author for correspondence: S Lindquist, Department of Neurology II, Otto-von-Guericke University, Leipziger Str. 44, 39120 Magdeburg, Germany. E-mail: sabine.lindquist@medizin.uni-magdeburg.de Received 11 April 2006; accepted 4 August 2006
} 
during Wallerian degeneration of axons [8,9], there is some support for the argument that MTR can be used as a marker for myelin destruction. The MTR is greatly reduced in chronic MS lesions, but less so in experimental autoimmune encephalomyelitis, where inflammation with only minor demyelination is the main finding [10]. Most convincingly, in pontine myelinolysis, a condition with 'pure' demyelination without inflammation and axonal damage, MTR is greatly reduced [11]. Furthermore, in patients with optic neuritis MTR is inversely correlated to the latency of visually evoked potentials, an electrophysiological indicator of demyelination [12].

MR diffusion imaging is sensitive to pathological changes in MS lesions [13-15], but the exact histopathological correlate is not clear [7]. Fractional anisotropy, a measure for the orientational dependence of diffusion, should monitor particularly well the integrity of white matter tracts which can be seen as oriented barriers of diffusion. Biochemical properties of tissue can be examined by ${ }^{1} \mathrm{H}$ magnetic resonance spectroscopy $\left({ }^{1} \mathrm{H}\right.$-MRS). $\mathrm{N}$-acetylaspartate (NAA) is almost exclusively contained in neuronal cell bodies and axons. A reduction of NAA has been demonstrated in the acute and chronic lesions $[6,16-19]$ of MS patients as well as in normal appearing white matter (NAWM) $[18,20-22]$. Importantly, the degree of NAA reduction seems to correlate with disability [22-25]. Concentrations of free choline (Cho) increase when membrane turnover is accelerated [26]. This occurs during inflammation, demyelination, gliosis and even remyelination. As a marker of acute inflammation, a rise in lactate can be measured within active lesions [16].

In order to more clearly define the pathological processes represented by MTR, DTI and ${ }^{1} \mathrm{H}-\mathrm{MRS}$, direct comparison of these parameters and correlation with histopathology is needed.
We present here a case of Balo's concentric sclerosis with lesion histopathology and serial MRI and MRS up to 13 months after the biopsy, providing, to date, the most detailed magnetic resonance and histology-based description of the evolution of such a lesion.

\section{Methods}

\section{Patient}

An open brain biopsy was taken from a 26 -year-old male, 24 days after the first clinical presentation. Tissue specimens were immediately fixed in formaldehyde and embedded in paraffin.

\section{Histopathology and immunohistochemistry}

Paraffin-embedded tissue was sectioned into $4-\mu \mathrm{m}$ slices, which were stained with haematoxylin and eosin (H\&E), Luxol-fast blue/periodic acid-Schiff LFB/PAS for myelin, as well as Bielschowsky's silver impregnation for axons. Immunohistochemical staining was performed with a biotin-avidin or an alkaline phosphatase/anti-alkaline phosphatase technique. Primary antibodies, their source, specificity and dilution, are listed in Table 1. Primary antibodies were omitted in controls. In situ hybridization, using digoxigenin-labeled riboprobes specific for proteolipid protein (PLP) mRNA, was carried out as previously described [27].

\section{Lesion staging}

The lesion was staged for its demyelinating activity according to recently established criteria [4]. Active demyelinating lesions, characterized by infiltrating macrophages positive for myelin degradation products, can be distinguished from inactive, comple-

Table 1 Primary antibodies

\begin{tabular}{llll}
\hline Specificity & Reactivity & Dilution & Source \\
\hline KiM1P (mo) & Macrophages/microglia [30] & $1: 20$ & $\begin{array}{l}\text { Professor HJ Radzun, Department of Pathology, } \\
\text { Göttingen, Germany }\end{array}$ \\
CD3 (mo) & T lymphocytes & $1: 200$ & $\begin{array}{l}\text { Serotec, Oxford, UK } \\
\text { Dako A/S, Glostrup, Denmark }\end{array}$ \\
CD8 (mo) & Cytotoxic T lymphocytes & $1: 50$ & Dako \\
GFAP (mo) & Gliosis & $1: 50$ & Chemicon International, Temecula, CA, USA \\
APP (mo) & Acute axonal damage & $1: 100$ & Dako \\
MBP (po) & Myelin basic protein & $1: 500$ & Sternberger Monoclonals Inc., Lutherville, USA \\
CNPase (mo) & 2,3'-Cyclic nucleotide 3'-phosphodiesterase & $1: 200$ & Professor Linington, Aberdeen, UK \\
MOG (mo) & Myelin oligodendrocyte glycoprotein & $1: 1000$ & Professor Richter-Landsberg, Oldenburg, Germany \\
MAG (mo) & Myelin-associated glycoprotein & $1: 1000$ & Serotec \\
PLP (mo) & Proteolipid protein & $1: 500$ & \\
\hline
\end{tabular}

mo, Monoclonal; po, polyclonal. 
tely demyelinated lesions and remyelinated lesions, in which uniformly thin, irregularly formed myelin sheaths are usually present.

\section{MR imaging and MR spectroscopy}

Written informed consent, according to the approval of the local ethics committee, was obtained from the patient before MR examinations.

Post-biopsy MR imaging and ${ }^{1} \mathrm{H}-\mathrm{MRS}$ were performed on a $1.5 \mathrm{~T}$ General Electrics Signa Horizon LX neuro-optimized MR tomograph (GE, Milwaukee, WI). A diffusion-sensitized echo planar imaging sequence ( $b$ value $1000 \mathrm{~s} / \mathrm{mm}^{2}$, TE $70 \mathrm{~ms}$ ) was applied to measure 39 axial slices (slice thickness 3 $\mathrm{mm}$, FOV $28 \mathrm{~cm}$, matrix $128 \times 128$ ) covering the whole cerebrum. Acquisitions without diffusionweighting and with 12 non-collinear orientations of the diffusion gradient were performed (four averages per orientation), each measured additionally with inverted gradient polarity (another four averages), enabling eddy current correction [28]. After additional correction for head movements, diffusion tensors were computed and apparent diffusion coefficient (ADC) and fractional anisotropy (FA) maps calculated.

MTI was performed based on a proton-density (PD)-weighted spin echo sequence (TR $2600 \mathrm{~ms}$, TE $20 \mathrm{~ms}$, one average, $256 \times 256,48$ slices, FOV 25 $\mathrm{cm}$ ) with and without MT saturation pulses. After correction for head movements, MTR maps were calculated.

Single-voxel ${ }^{1} \mathrm{H}$-MRS used point-resolved spectroscopy (PRESS) with TR $1500 \mathrm{~ms}$, TE $135 \mathrm{~ms}$ and 256 averages. In total, three voxels were outlined: (1) in the central lesion area, including the position of tissue extraction; (2) in an area superior to the biopsy, including solely lesioned tissue but excluding the biopsy region itself; and (3) in the NAWM contralateral to the location of the biopsy. During follow-up, exact repositioning of the voxels was achieved by manual alignment relative to anatomical landmarks.

The acquired data were analysed using LCModel (Vers. 6.1.0) [29]. Concentrations are given in arbitrary units.

The 'conventional' structural MR images depicted in Figure 1 were obtained for diagnostic purposes on a 1.5 T Siemens Magnetom Vision MR tomograph, and have not been used for any quantitative analysis.

\section{Coregistration and segmentation of regions of interest}

All coregistration tasks were processed using SPM2 (Wellcome Department of Cognitive Neurology,
Institute of Neurology, London, UK). Accurate anatomical matching (Figure 4) of ADC, FA and MTR maps across all follow-up examinations was accomplished by linear and non-linear transformations. The $\mathrm{T}_{2}$-weighted fast spin echo (FSE) images of the second examination time-point served as template for all coregistrations.

Segmentation of the lesion was motivated by their dissimilar appearance, presumably due to different histopathological processes. Three ROIs were defined on MTR maps: (1) the region of the biopsy channel and tissue extraction itself; (2) a central region that featured relatively high MTR values; (3) a ring-shaped low-MTR region surrounding the region 2. Additionally, a fourth ring-shaped region, inconspicuous on MTR maps, was defined by its hyperintensity on protondensity-weighted maps. Segmentations were performed using DispImage (Dave Plummer, University College London, UK). Any overlap of the ROIs was avoided.

\section{Results}

\section{Case report}

A 26-year-old male of Italian descent presented with an 11-day history of fluctuating right-sided hemiparesis. Examination disclosed a discrete right-sided facial weakness and spastic hemiparesis, with a grade $4 / 5$ weakness in the upper, grade $4+/ 5$ in the lower extremity, mild hemihypaesthesia on the right, and some slight difficulty in finding words (Expanded Disability Status Scale; EDSS: 3.0). Cranial MRI revealed a left hemispheric paraventricular oval lesion with Gdenhancement. An additional small lesion, $2 \mathrm{~mm}$ in diameter, was seen in the right frontotemporal region. Lumbar puncture showed 3 cells $/ \mu \mathrm{L}$, slightly raised protein at $612 \mathrm{mg} / \mathrm{L}$, and normal glucose and lactate. The IgG-Index was not raised (0.5), but oligoclonal bands were positive at this point; a repeat examination after six months was negative.

Additional clinical investigations did not show any evidence for malignancy or systemic autoimmune diseases.

A further cranial MRI, 16 days after onset of symptoms, showed that the lesion had increased to $2 \mathrm{~cm}$ in diameter and was still Gd-enhancing (Figure 1A). As the appearance of this lesion could resemble a lymphoma, abscess or autoinflammatory process, a stereotactic biopsy was performed on day 25 after onset of symptoms. Immediate histopathology showed an inflammatory demyelinating process. A few hours after the biopsy, the patient's clinical condition worsened to hemiplegia, severe 


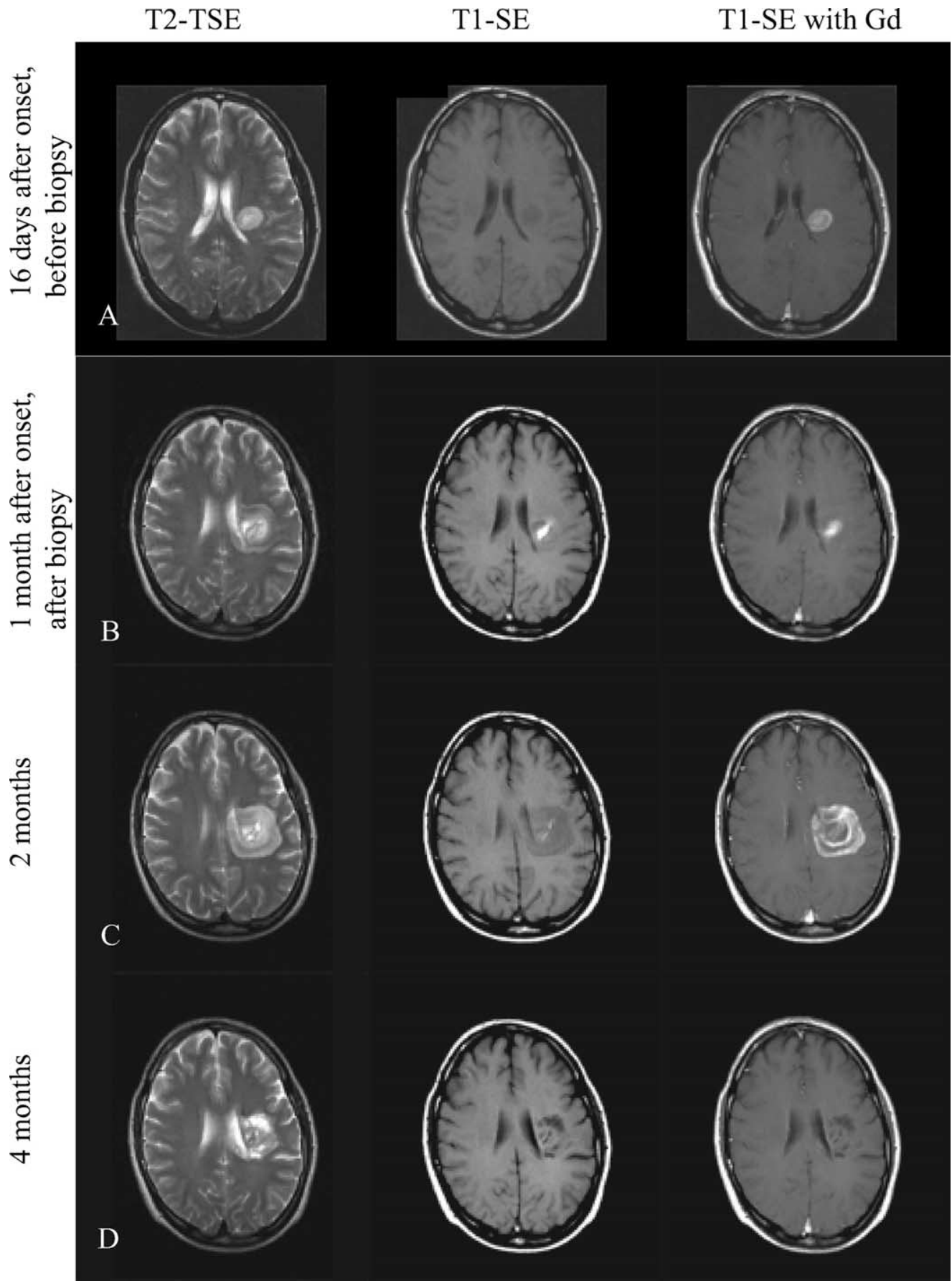

Figure 1 Series of an axial section of $T_{2^{-}}$as well as $T_{1}$-weighted MR images with and without Gd-DTPA contrast. (A) In the examination before the biopsy an oval lesion can be demonstrated, which is hyointense in $T_{1}$ and hyperintense in $T_{2}$. The core appears slightly more $\mathrm{T}_{2}$-hyperintense than the remaining lesion. With $\mathrm{Gd}$ contrast, the core and the rim of the lesion are enhanced. (B) At one month after onset of symptoms and seven days after stereotaxic biopsy, the site of biopsy can be distinguished clearly in the core of the lesion, small amounts of blood appear hyperintense on $T_{1}$. The $T_{2}$-weighted image shows two hyperintense rings. The center of the lesion not covered by blood is clearly hypointense in $T_{1}$, the periphery is slightly hypointense. There is no Gd-enhancement. Note that the patient was under treatment with high dose corticosteroids at this time. (C) At two months, $T_{2}$ hyperintensity of at least two concentric rings is greatly increased. On $T_{1}$, several rings of hypo-and hyperintensity can be distinguished. Gadolinium-contrast shows two simultaneous rings of strong enhancement. (D) At four months, the lesion has shrunk. Concentric rings are not readily distinguishable on $T_{2}$. On $T_{1}$ the biopsy site is hypointense, the surrounding core area hyperintense and the periphery is severely decreased in intensity. There is no longer gadolinium-enhancement. 
hemihypaesthesia, facial weakness, deviation of the tongue to the right, apraxia for motor and language tasks with total inability to speak, as well as a pronounced impairment of speech comprehension (EDSS: 8.5). The patient was treated immediately with high doses of iv methylprednisolone: $1 \mathrm{~g}$ /day for six days, then $2 \mathrm{~g}$ /day for three days, followed by iv immunoglobulins $30 \mathrm{~g} /$ day for five days. Due to unresponsiveness, after a treatment pause of 10 days, the patient received five courses of plasmapheresis within 14 days. The slow but profound clinical improvement (illustrated by the changes in EDSS in Figure 4) could not obviously be related to any immunosuppressive treatment. However, these therapies may have halted a very aggressive disease course.

The evolution of the inflammatory lesion was monitored for therapeutic purposes by conventional MRI. At one month, the lesion was edematous and about $4 \mathrm{~cm}$ in diameter, extending into the internal capsule, the lateral thalamus and temporal insular cortex. Gd-enhancement at this point was most probably reduced by the treatment with iv steroids (Figure 1B). At two months, the lesion size was only slightly increased on $T_{2^{-}}$ weighted images, but $\mathrm{T}_{1}$-weighting showed two hypointense rings alternating with hyperintense rings (Figure 1C). Gd-contrast clearly demonstrated two distinct rings of enhancement. After four months, MRI showed a reduction in lesion size, some enlargement of the left ventricle towards the lesion site, no edema and no Gdenhancement (Figure 1D).

\section{Neuropathology}

Neuropathology revealed an inflammatory demyelinating process (Figure 2). The $H \& E$ staining showed a hypercellular lesion with perivascular lymphocytic infiltrates, macrophages and severe edema. Immunocytochemistry identified the infiltrating lymphocytes as CD3- and CD8-positive T cells. There were numerous KiM1P-positive macrophages/microglia present within the lesion (Figure 2D-F). LFB/PAS myelin staining and immunohistochemistry for myelin proteins revealed active demyelination as LFB-positive degradation products (not shown), and all tested myelin proteins could be demonstrated in the macrophage cytoplasm (shown for MBP in Figure 2A) indicating early active, recent demyelination. Oligodendrocytes were significantly reduced in number and there were some MAG- or CNPase-positive oligodendrocytes with condensed nuclei present, indicative of apoptosis (Figure 2B,C). These active demyelinating areas clearly followed an immunopathological pattern III, as previously described [4].
Besides the active demyelinating areas, parts of the lesion were completely demyelinated, with no signs of ongoing myelin breakdown or remyelination. Most strikingly, axonal density was significantly reduced (Figure 2G,H) and massive acute axonal damage was demonstrated by immunoreactivity against APP (Figure 2I).

\section{${ }^{1}$ H-MR spectroscopy}

To study whether there are differences in pathological processes in the periphery of the large lesion compared to the core, we measured metabolite changes in one voxel containing the biopsied site (biopsy-voxel) and another voxel directly above the core voxel (Figure 3A).

In both voxels (Figure 3B,C) a striking reduction of creatine $(\mathrm{Cr})$ compared to contralateral white matter (34.9 units) was evident. Creatine increased again over the next 13 months, but stayed greatly reduced compared to contralateral white matter. Cho was also diminished compared to contralateral white matter (14.7 units) in the biopsy-voxel and recovered, to some extent, during the observation period. In the voxel above the biopsy, Cho was reduced at three months and otherwise not altered. N-acetylaspartate (NAA) was massively reduced compared to contralateral white matter (68.3 units) and stayed low in the biopsy voxel, but increased slightly in the voxel above the biopsy. An increase of lactate was most pronounced in the voxel above the biopsy and at the initial examination at one month. Levels in both voxels decreased in the following two examinations and returned to not measurable levels, similar to the contralateral side, thereafter.

\section{Magnetization transfer imaging and diffusion tensor imaging}

To separately investigate the dynamics of pathological processes in the concentric rings typical for Balo's disease MTR maps were segmented as described (see Methods section and Figure 4A).

Quantification in these separate rings (Figure 4B) showed that MTR was most decreased at the initial two weeks after biopsy and five weeks after onset of symptoms in region 1 . In this region, MTR continuously increased over the following five months and then stabilized at $70-75 \%$ of the value of the contralateral side. MTR in region 2 was much higher than in region 1 at the first time point (69\% of the value of the contralateral side), but showed almost no change over time. Region 3 showed the greatest dynamics in MTR. Starting only slightly decreased with $88 \%$ of the contral- 

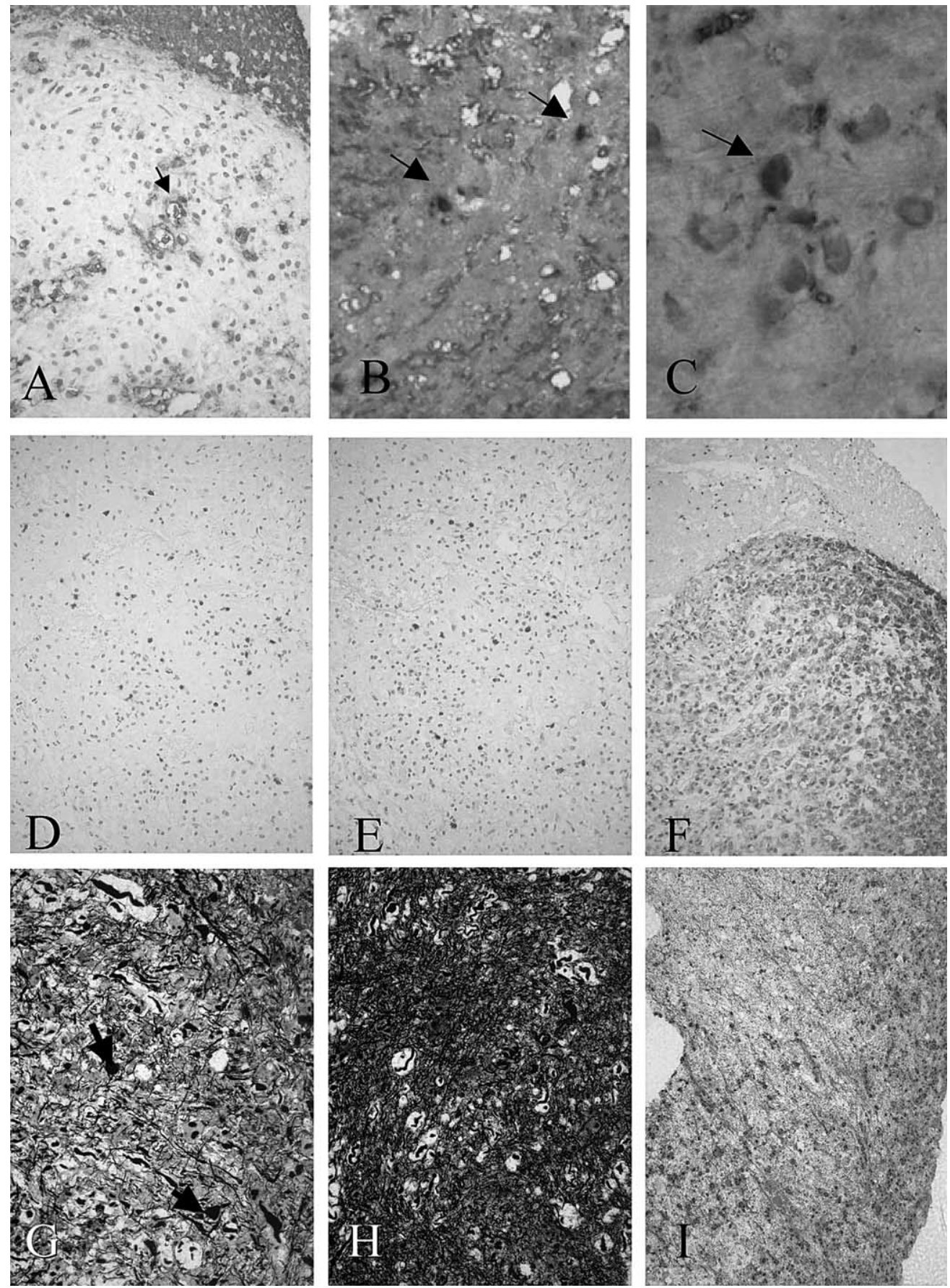

Figure 2 (A) Immunocytochemistry for myelin basic protein (MBP) (magnification $\times 400$ ): extensive demyelination with sharp border to the periplaques white matter. Within the demyelinated area, foamy macrophages exhibit MBP-positive myelin degradation products (arrow). (B) Detection of few oligodendrocytes (magnification $\times 200$ ) within the demyelinating lesion stained for PLP mRNA (black cells, arrows) and PLP protein (red); cell nuclei are counter-stained with hematoxylin (blue). (C) Immunocytochemistry for CNPase (magnification $\times 1000$ ): within the demyelinated area apoptotic oligodendrocytes with condensed nuclei immunopositive for CNPase can be demonstrated (arrow). (D) Immunocytochemistry for CD3 (magnification $\times 200$ ): diffuse infiltration of CD3-positive T-cells within the lesion. (E) Immunocytochemistry for CD8 (magnification $\times 200$ ): many of the infiltrating T-cells can be identified as CD8-positive cytotoxic T-lymphocytes. (F) Immunocytochemistry for Ki-M1P (magnification $\times 200$ ): diffuse and dense infiltration of foamy macrophages within the demyelinated area, as demonstrated with the pan-macrophage-marker Ki-M1P. (G) Bielschowsky's silver impregnation (magnification $\times 400$ ): extensive loss of axons as well as axonal spheroids (arrow) within the lesion. (H) Bielschowsky's silver impregnation (magnification $\times 200$ ): in contrast to the lesion, the periplaque white matter displays a homogeneously dense network of axons. (I) Immunocytochemistry for amyloid precursor protein (APP) (magnification $\times 200$ ): numerous APPpositive axonal spheroids indicate extensive axonal damage within the lesion. 
ateral side, it greatly decreased up to $46 \%$ three months after onset of symptoms and increased again over the following three months to stabilize at $74 \%$. Region 4 showed an almost normal MTR ( $95 \%$ of contralateral side) with a slight decrease at three months.

The mean ADC showed a mirror image of the changes in MTR over time (Figure 4B), only region 1 differed, showing stably increased mean ADC over the observation period. Quantification of the mean FA adds information to the MTR and ADC measurements (Figure 4B). FA in region 1 was continuously increasing from $48 \%$ of the value of the contralateral side one month after onset of symptoms to $86 \% 13$ months later. In region 2, FA showed a slight decrease at three months, but was otherwise stable. Interestingly, in region 3, FA was already greatly decreased in the initial examination at one month, when MTR and ADC still showed values closer to those of the contralateral side. In this region, FA was again lowest at 3 months, increased to $47 \%$ of the contralateral side at six months and stabilized thereafter. FA in region 4 showed the same dynamics as MTR and ADC, but was clearly abnormal with values of $70 \%$ (one month), $55 \%$ (three months) up to $80 \%$ (14 months) of the contralateral side.

\section{Discussion}

The diagnosis of Balo's concentric sclerosis was supported by the pathognomonic radiological appearance of a single lesion with alternating rings of hypo- and hyperintensity in $\mathrm{T}_{1}$-weighted images, and at least two rings of simultaneous Gd-enhancement [2,30-32]. Histology revealed active demyelination with oligodendrocyte apoptosis representing immunopathological pattern III[4], which is characteristic for Balo's concentric sclerosis [3]. Other, probably more central lesional areas, showed inactive demyelination with significant axonal loss. Matching the diagnosis of Balo's concentric sclerosis was the young age of the patient, the acute onset and rapid progression as well as the monophasic course of the disease, which is also indicated by the lacking persistence of oligoclonal bands. Balo's disease has been described as progressive and frequently fatal. However, self-limiting cases have been monitored with MRI. Serial imaging in selected cases of Balo's disease showed the centrifugal development of enhancing rings, presumably representing ongoing inflammation. These $\mathrm{T}_{1}$ - and $\mathrm{T}_{2}$-isointense rings became $\mathrm{T}_{1}$-hypointense on follow-up, indicating sequential demyelination and potential axon damage $[30,31$.
${ }^{1} \mathrm{H}-\mathrm{MRS}$ corresponds to substantial loss of axons and myelin seen in histology and indicates a failure of local repair

The biopsy demonstrated massive tissue destruction, which most likely accounts for the $60-75 \%$ reduction of total $\mathrm{Cr}$ measured by ${ }^{1} \mathrm{H}$-MRS. We, therefore, represent metabolite concentrations as absolute values. Altered $\mathrm{Cr}$ levels in lesions [26] and NAWM [33] of MS patients have been described, and a relative increase in $\mathrm{Cr}$ has been interpreted as gliosis [33]. In context with other metabolites, the slow increase of Cr over 13 months in our case is likely to also indicate gliosis. The striking finding in the biopsy, an approximately $80 \%$ axonal loss, corresponded well to a reduction of NAA by 80 $90 \%$. This axonal loss appeared to be largely permanent. Only in the more peripheral voxel above the biopsy, a slight increase in NAA over 10 months could be monitored. This may either represent shrinking of the lesioned tissue and inclusion of unaffected tissue into this voxel or may indicate limited repair. NAA is a well-studied indicator of axonal integrity and was correlated to axonal density in biopsied MS patients [34]. Decreased NAA or NAA/Cr were also found in selected cases of Balo's concentric sclerosis; one of these was confirmed by biopsy [35-37]. Contrary to these previous reports, where increased Cho was thought to represent higher membrane turnover, Cho concentrations were reduced in the lesion centre in our case. This may be explained by the biopsied tissue, which showed substantial loss of membranous structure and apoptosis of oligodendrocytes. Immunological pattern III identified in our case is generally associated with pronounced death of oligodendrocytes via apoptosis and subsequently remyelination is in general very sparse. One may speculate that decreased Cho, which is a marker for membrane turnover, could thus be a negative prognostic factor for repair. Over the next year, Cho levels recovered to some extent, indicating limited tissue reorganization. Interestingly, in the more peripheral voxel, Cho was normal initially, but decreased at three months, thus demonstrating in vivo the centrifugal extension of pathogenic processes. Correspondingly, increased lactate, particularly in the periphery of the lesion, persisting for four months indicates ongoing inflammation, as lactate is thought to represent anaerobic glycolytic metabolism in activated leucocytes infiltrating the lesion [38]. It cannot be excluded that the surgical trauma contributed to some of the metabolite changes observed in the core voxel, however, the magnitude of $\mathrm{Cr}$, NAA and Cho reduction cannot be solely explained by lesioning a very small proportion of the voxel volume. 


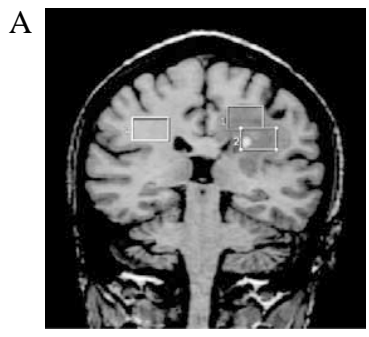

Voxel 1: biopsy

Voxel 2: above biopsy

Voxel 3: contralateral
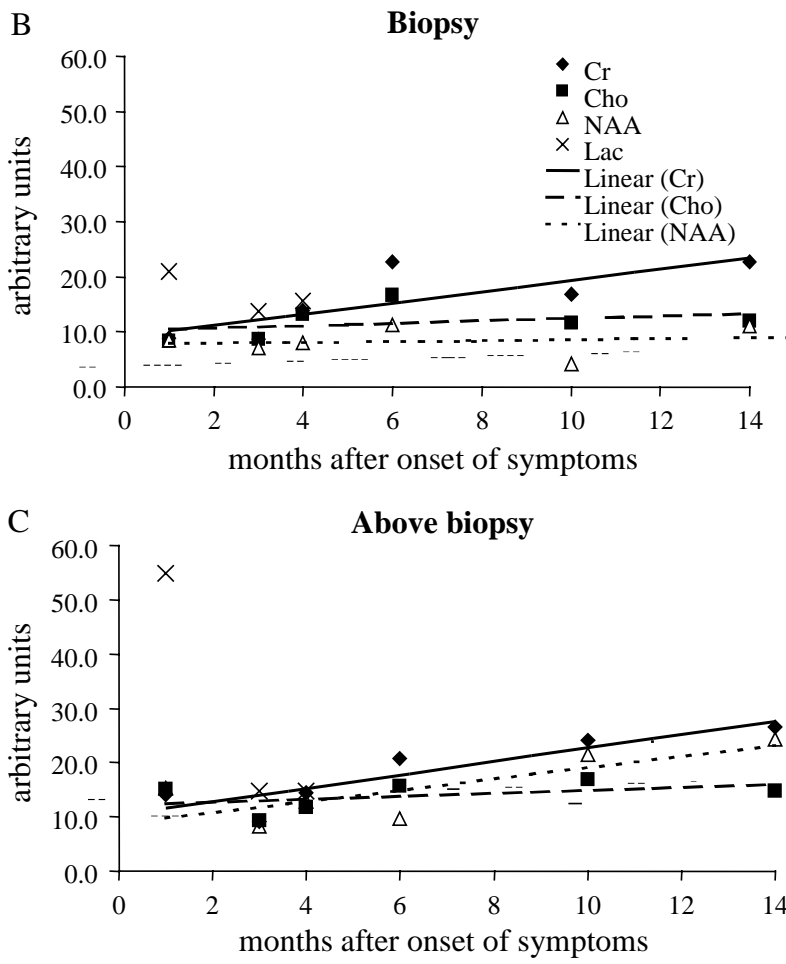

Figure $3{ }^{1} \mathrm{H}-\mathrm{MR}$ spectroscopy was carried out in three different voxels (4.8 mL each), which were defined on axial FLAIR images, and are depicted in $(A)$ on $T_{1}$-weighted images from a three-dimensional data set. Metabolite concentrations within a voxel containing the biopsied site (B), as well as a voxel above the biopsied site (C), are shown at different examination time points. Given are the absolute values in arbitrary units. Linear regression curves were fitted for NAA, Cr and Cho. Lactate could only be measured at the first three time points and the decline is not linear. Data are compared with absolute concentrations from the contrary white matter (Voxel 3), which are given in the text. NAA, Cr and $\mathrm{Cho}$ are greatly reduced in both voxels containing the lesion. $\mathrm{Cr}$ is increasing over time, Cho stays constant. NAA stays constant in the voxel containing the biopsy, but increases steadily in the voxel above the biopsy. Lactate is slightly increased in the voxel containing the biopsy, but massively elevated in the more peripheral voxel at the first examination time point and decreases thereafter. $\mathrm{Cr}$, creatine; Cho, choline; NAA, N-acetylaspartate; Lac, lactate.

Decreased MTR and increased diffusion monitor sequential tissue destruction in selected lesion areas

Whereas the interpretation of spectroscopy results is hampered by the need for relatively large voxels, thereby averaging pathological changes, a much higher spatial resolution can be achieved in MT and DT imaging. This allows the segmentation of the lesion into rings that are typical for Balo's and to look at pathological processes within these rings separately. Reduced MTR can demonstrate demyelination [12], but more generally represents a decreased amount of macromolecular structure per unit volume [39]. The massive reduction of MTR in the core of the lesion (region 1) in our case therefore agrees with the tissue destruction seen in histology and may also represent some damage caused by the biopsy. It cannot differentiate axonal damage and demyelination, as both are present. In region 2, higher MTR suggests less tissue disintegration. The stability of the moderate reduction over the whole observation period indicates that neither disease nor repair processes were active in this area. Evidence for the hypothesis of centrifugal extension of disease processes in Balo's concentric sclerosis comes from comparison with the MTR dynamics in region 3. Two weeks after the biopsy, which showed active demyelination in more peripheral areas and marked infiltration of macrophages and T lymphocytes, MTR was only slightly reduced. An extensive fall in MTR was only seen two months later. Given the fact that at the time of the first examination, lactate concentrations were high particularly in the periphery, the high MTR in region 3 at this time may not represent almost intact tissue, but rather an area densely filled with inflammatory cells. The second examination may pick up subsequent tissue damage caused by these cells. Between three and six months after onset of symptoms, MTR increased steadily, representing reorganization of the tissue. In region 4 , a slight reduction of MTR only at three months would suggest the predominance of edema without substantial demyelination and axonal loss [11].

ADC measurements mirror the changes of MT ratios for the most part. This overlap in information is expected, as MTR is sensitive to the density of macromolecules, which also form barriers to free diffusion of water.

Diffusion tensor imaging allows the distinction of early and late changes from normal white matter

Importantly, while tissue destruction in MS lesions is well monitored by decreased MTR and increased diffusivity $[13,15,40]$, acute and chronic pathogenic changes are not [15]. This discrepancy is caused by the additive effects of different pathogenic changes: edema, demyelination and axonal loss lead to an increase [15,41-43], high cellular density either due to proinflammatory cells or gliosis, decreases 
A

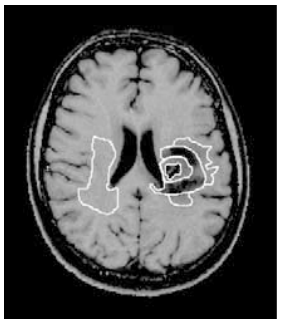

MTR map

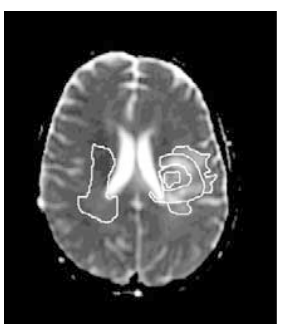

ADC map

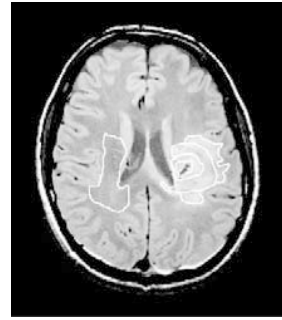

proton density map

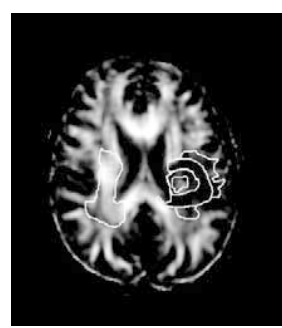

FA map

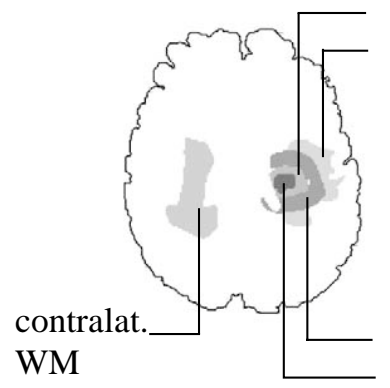

region 2

region 4

region 3

region 1
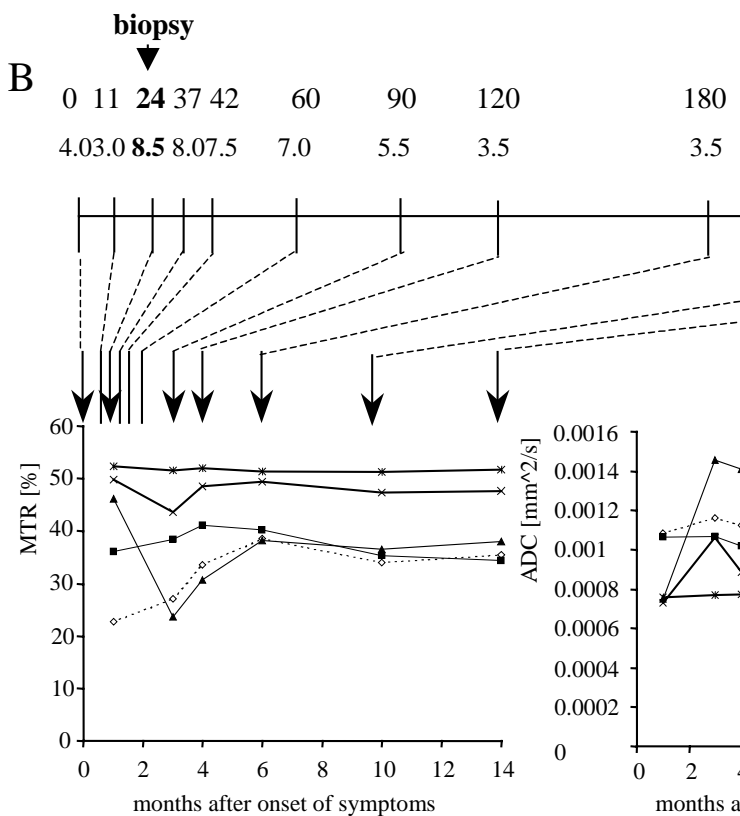

80

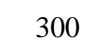

days

520

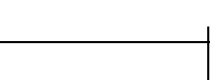

$3.5-2.5$

EDSS

2.5
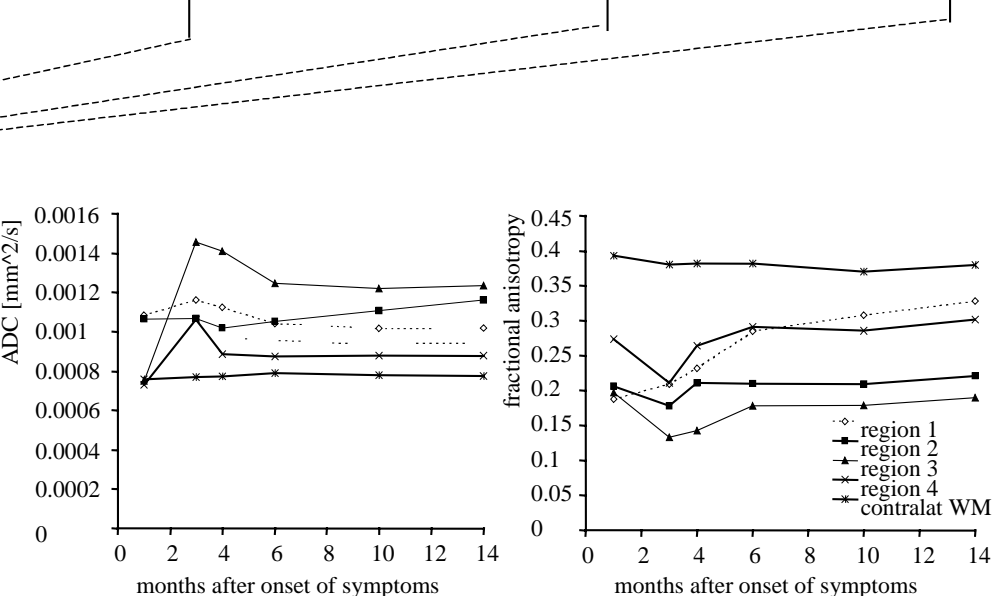

Figure 4 (A) Representative axial sections of the MTR and proton density maps where regions of interest were defined according to the schematic picture (see also Materials and methods); ADC and FA maps which these regions were transferred on. (B) Magnetization transfer ratio (MTR), apparent diffusion coefficient (ADC) and fractional anisotropy (FA) of specific regions of interest within the lesion for different examination time points. Given are the means of all slices containing these regions. For comparison with the clinical course, EDSS scores for the respective time points are given. Large arrows indicate the onset of symptoms and time points for MRI examinations, small arrow indicate the time of biopsy.

diffusivity $[13,44]$. Fractional anisotropy, a measure of directionality of tissue, is also decreased in disintegrated parenchyma with increased diffusivity. However, our case demonstrates that FA is more sensitive than MTR and ADC to early inflammatory changes. FA most clearly depicts pathological changes in the periphery (region 4 ), where accord- ing to MRS and by extrapolating the biopsy findings, inflammation is ongoing. Indeed, Werring et al., already demonstrated that FA is more sensitive than ADC to the acuity of the lesion [15]. Furthermore, in region 3, FA shows clearly decreased values from the first examination when MTR and ADC are still close to normal. The 
comparison of FA with MTR strongly supports our earlier conclusion, that at this time point region 3 is not unaffected, but rather filled with inflammatory cells. While increasing MTR and decreasing diffusivity at later examinations indicate reorganization, a consistently lowered FA demonstrates that directionality is not re-established. This clearly indicates gliosis as the cause of increased tissue density. The weak correlation between ADC and FA in MS lesions $[15,45]$ and the lack thereof between FA and MTR in corpus callosum of MS patients [45] clearly demonstrates that complementary information can be gained from combining MTI and DTI.

\section{Recovery despite the lack of local repair}

Rapid development of the lesion prior to biopsy and massive tissue destruction shown histopathologically demonstrate an aggressive inflammatory process typical for Balo's disease. Although the dramatic worsening of symptoms soon after biopsy suggests an either direct or indirect effect of the stereotactic procedure, it is unlikely that the relatively small trauma from biopsying the already severely demyelinated area with minimal axonal preservation caused significant additional damage. We rather think that the observed rapid enlargement of the lesion into the internal capsule and subcortical areas disrupting efferents of Broca's area, possibly triggered by blood-brain barrier damage following biopsy, caused this extensive pyramidal tract damage and aphasia. Indeed, there is evidence from experimental studies that neuropathological damage is augmented in immunized animals after trauma [46]. Acute inflammatory processes may also better explain that the peak of clinical symptoms preceeded the peak of tissue destruction. Mediators, such as nitric oxide or small peptides, may have caused extensive conduction blocks [47-49] and edema may have further compromised axonal function. Therefore, recovery may have resulted from resolving inflammation in the periphery of the lesion, whereas the functionally less important centre failed to repair. Additionally or alternatively, neuronal plasticity may have allowed new circuits to form in this young patient.

\section{Conclusion}

A multimodal MRI approach enables us to follow tissue changes in a demyelinating inflammatory lesion. Specifically, we can demonstrate areas with apparently normal MTR showing pathological changes in DTI. The combination with DTI and MRS together with the temporal pattern of lesion development serve to contextually interpret MTR values. While the initial observations appear encouraging, the applicability of these parameters combined to monitor the acuity and the fate of an evolving MS lesion, requires further evaluation.

\section{Acknowledgements}

This work was supported by a BMBF Grant (01GO0504) and, in part, by an unrestricted research grant from Serono GmbH Germany.

\section{References}

1. Karaarslaan E, Altintas A, Senol U, Yeni N, Dincer A, Bayindir C et al. Balo's concentric sclerosis: clinical and radiologic features of five cases. Am J Neuroradiol 2001; 22: 1362-67.

2. Kastrup O, Stude P, Limmroth V. Balo's concentric sclerosis. Evolution of active demyelination demonstrated by serial contrast-enhanced MRI. J Neurol 2002; 249: $811-14$.

3. Stadelmann C, Ludwin S, Tabira T, Guseo A, Lucchinetti CF, Leel-Ossy $\mathbf{L}$ et al. Tissue preconditioning may explain concentric lesions in Balo's type of multiple sclerosis. Brain 2005; 128: 979-87.

4. Lucchinetti C, Brüick W, Parisi J, Scheithauer B, Rodriguez M, Lassmann H. Heterogeneity of multiple sclerosis lesions: implications for the pathogenesis of demyelination. Ann Neurol 2000; 47: 691-93.

5. Brüick W, Bitsch A, Kolenda H, Brüick Y, Stiefel M, Lassmann H. Inflammatory central nervous system demyelination: correlation of magnetic resonance imaging findings with lesion pathology. Ann Neurol 1997; 42: $783-93$.

6. Gass A, Barker GJ, Kidd D, Thorpe JW, Mac Manus D, Brennan A et al. Correlation of magnetisation transfer ratio with clinical disability in multiple sclerosis. Ann Neurol 1994; 36: 62-67.

7. Mottershead JP, Schmierer K, Clemence M, Thornton JS, Scaravilli F, Barker GJ et al. High field MRI correlates of myelin content and axonal density in multiple sclerosis - a post-mortem study of the spinal cord. J Neurol 2003; 250: $1293-301$.

8. Lexa FJ, Grossman RI, Rosenquist AC. MR of Wallerian degeneration in the feline visual system: characterization by magnetisation transfer rate with histopathologic correlation. Am J Neuroradiol 1994; 15: 201-12.

9. Van Waesberghe JH, Kamphorst $\mathbf{W}$, De Groot $\mathbf{C J}$, van Walderveen MA, Castelijns JA, Ravid $R$ et al. Axonal loss in multiple sclerosis lesions: magnetic resonance imaging insights into substrates of disability. Ann Neurol 1999; 46: 747-54.

10. Dousset V, Grossman RI, Ramer KN, Schnall MD, Young LH, Gonzalez-Scarano F et al. Experimental allergic encephalomyelitis and multiple sclerosis: lesion characterisation with magnetisation transfer imaging. Radiology 1992; 182: 483-91.

11. Davie CA, Silver NC, Barker GJ, Tofts PS, Thompson AJ, McDonald WI et al. Does the extent of axonal loss and demyelination from chronic lesions in multiple sclerosis correlate with the clinical subgroup? J Neurol Neurosurg Psychiatry 1999; 67: 710-15.

12. Thorpe JW, Barker GJ, Jones SJ, Moseley I, Losseff N, MacManus DG et al. Magnetisation transfer ratios and transverse magnetisation decay curves in optic neuritis: correlation with clinical findings and electro- 
physiology. J Neurol Neurosurg Psychiatry 1995; 59: 487 92.

13. Christiansen P, Gideon $P$, Thomsen C, Stubgaard M, Henriksen O, Larsson HB. Increased water selfdiffusion in chronic plaques and in apparently normal white matter in patients with multiple sclerosis. Acta Neurol Scand 1993; 87: 195-99.

14. Horsfield MA, Larsson HB, Jones DK, Gass A. Diffusion magnetic resonance imaging in multiple sclerosis. J Neurol Neurosurg Psychiatry 1998; 64: S80-84.

15. Werring DJ, Clark CA, Barker GJ, Thompson AJ, Miller DH. Diffusion tensor imaging of lesions and normal-appearing white matter in multiple sclerosis. Neurology 1999; 52: 1626-32.

16. Matthews PM, Francis G, Antel J, Arnold DL. Proton magnetic resonance spectroscopy for metabolic characterization of plaques in multiple sclerosis. Neurology 1991; 41: $1251-56$.

17. Miller DH, Austin SJ, Connelly A, Youl BD, Gadian DG, McDonald WI. Proton magnetic resonance spectroscopy of an acute and chronic lesion in multiple sclerosis. Lancet 1991; 337: 58-59.

18. Davie CA, Hawkins CP, Barker GJ, Brennan A, Tofts PS, Miller DH et al. Serial proton magnetic resonance spectroscopy in acute multiple sclerosis lesions. Brain 1994; 117: 49-58.

19. De Stefano N, Matthews PM, Antel JP, Preul M, Francis G, Arnold DL. Chemical pathology of acute demyelinating lesions and its correlation with disability. Ann Neurol 1995; 38: 901-909.

20. Husted CA, Goodin DS, Hugg JW, Maudsley AA, Tsuruda JS, de Bie SH et al. Biochemical alterations in multiple sclerosis lesions and normal-appearing white matter detected by in vivo $31 \mathrm{P}$ and ${ }^{1} \mathrm{H}$ spectroscopic imaging. Ann Neurol 1994; 36: 157-65.

21. Narayanan S, Fu L, Pioro E, De Stefano N, Collins DL, Francis GS et al. Imaging of axonal damage in multiple sclerosis: spatial distribution of magnetic resonance imaging lesions. Ann Neurol 1997; 41: 385-91.

22. Fu L, Matthews PM, De Stefano N, Worsley KJ, Narayanan S, Francis GS et al. Imaging axonal damage of normal-appearing white matter in multiple sclerosis. Brain 1998; 121: 103-13.

23. Davie CA, Barker GJ, Webb S, Tofts PS, Thompson AJ, Harding AE et al. Persistent functional deficit in multiple sclerosis and autosomal dominant cerebellar ataxia is associated with axon loss. Brain 1995; 118: $1583-92$.

24. Davie CA, Barker GJ, Thompson AJ, Tofts PS, McDonald WI, Miller DH. ${ }^{1} \mathrm{H}$ magnetic resonance spectroscopy of chronic cerebral white matter lesions and normal appearing white matter in multiple sclerosis. $J$ Neurol Neurosurg Psychiatry 1997; 63: 736-42.

25. Matthews PM, Pioro E, Narayanan S, De Stefano N, Fu L, Francis $\mathbf{G}$ et al. Assessment of lesion pathology in multiple sclerosis using quantitative MRI morphometry and magnetic resonance spectroscopy. Brain 1996; 119: $715-22$.

26. Li BSY, Regal J, Soher BJ, Mannon LJ, Grossman RI, Gonen 0 . Brain metabolite profiles of $T_{1}$-hypointense lesions in relapsing-remitting multiple sclerosis. $\mathrm{Am}$ J Neuroradiol 2003; 24: 68-74.

27. Lucchinetti C, Brück W, Parisi J et al. A quantitative analysis of oligodendrocytes in multiple sclerosis lesions. A study of 117 cases. Brain 1999; 122: 2279-95.

28. Bodammer N, Kaufmann J, Kanowski M, Tempelmann C. Eddy current correction in diffusion-weighted imaging using pairs of images acquired with opposite diffusion gradient polarity. Magn Reson Med 2004; 51: $188-93$.
29. Provencher, SW. Estimation of metabolite concentrations from localized in vivo proton NMR spectra. Magn Reson Med 1993; 30: 672-79.

30. Sekijima Y, Tokuda T, Hashimoto T, Koh CS, Shoji S, Yanagisawa N. Serial magnetic resonance imaging (MRI) study of a patient with Balo's concentric sclerosis treated with immunadsorption plasmapheresis. Mult Scler 1997; 2: 291-94.

31. Chen CJ, Nai-Shin C, Lu CS, Sung CY. Serial magnetic resonance imaging in patients with Balo's concentric sclerosis: natural history of lesion development. Ann Neurol 1999; 46: 651-56.

32. Caracciolo JT, Murtagh RD, Rojiani AM, Murtagh FR. Pathognomonic MR imaging findings in Balo's concentric sclerosis. Am J Neuroradiol 2001; 22: 292-93.

33. Vrenken H, Barkhof F, Uitdehaag BM, Castelijns JA, Polman CH, Pouwels PJ. MR spectroscopic evidence for glial increase but not for neuro-axonal damage in MS normal-appearing white matter. Magn Reson Med 2005; 53: 256-66.

34. Bitsch A, Bruhn H, Vougioukas V, Stringaris A, Lassmann H, Frahm J et al. Inflammatory CNS demyelination: histopathologic correlation with in vivo quantitative proton MR spectroscopy. Am J Neuroradiol 1999; 20: $1619-27$.

35. Kim MO, Lee SA, Choi CG, Huh JR, Lee MC. Balo's concentric sclerosis: a clinical case study of brain MRI, biopsy, and proton magnetic resonance spectroscopic findings. J Neurol Neurosurg Psychiatry 1997; 62: 655-58.

36. Chen CJ. Serial proton magnetic resonance spectroscopy in lesions of Balo concentric sclerosis. J Comput Assist Tomogr 2001; 25: 713-18.

37. Bruneteau G, Guillevin R, Tourbah A, Papeix C, Chiras J, Pierrot-Deseilligny C. [Contribution of proton magnetic resonance spectroscopy to the diagnosis of Balo's concentric sclerosis]. Rev Neurol (Paris) 2005; 161: $455-58$.

38. Simone IL, Federico F, Trojano M, Tortorella C, Liguori M, Giannini P et al. High-resolution proton MR spectroscopy of cerebrospinal fluid in MS patients. Comparison with biochemical changes in demyelinating plaques. J Neurol Sci 1996; 144: 182-90.

39. Wolff SD, Balaban RS. Magnetisation transfer contrast (MTC) and tissue water proton relaxation in vivo. Magn Reson Med 1989; 10: $135-44$.

40. Larsson HB, Thomsen C, Frederiksen J, Subgaard M, Henriksen o. In vivo magnetic resonance diffusion measurement in patients with multiple sclerosis. Magn Reson Imaging 1992; 10: 7-12.

41. Barnes D, Munro PMG, Youl BD, Primeas W, McDonald WI. The longstanding MS lesion: a quantitative MRI and electron microscopy study. Brain 1991; 114: $1271-80$.

42. Heide AC, Richards TL, Alvord EC, Peterson J, Rose LM. Diffusion imaging of experimental encephalomyelitis. Magn Reson Med 1993; 29: 478-84.

43. Verhoye MR, Gravenmade EJ, Raman ER, Van Reemts J, Van der Linden A. In vivo non-invasive determination of abnormal water diffusion in the rat brain studied in an animal model for multiple sclerosis by diffusion-weighted NMR imaging. Magn Reson Imaging 1996; 14: $521-32$.

44. Gass A, Niendorf T, Hirsch, JG. Acute and chronic changes of the apparent diffusion coefficient in neurological disorders - biophysical mechanisms and possible underlying histopathology. J Neurol Sci 2001; 186: S1523.

45. Coombs BD, Best A, Brown MS, Miller DE, Corboy J, Baier $\mathbf{M}$ et al. Multiple sclerosis pathology in the normal and abnormal appearing white matter of the 
corpus callosum by diffusion tensor imaging. Mult Scler 2004; 10: 392-97.

46. Jones TB, Ankeny DP, Guan Z, McGaughy V, Fisher LC, Basso DM et al. Passive or active immunization with myelin basic protein impairs neurological function and exacerbates neuropathology after spinal cord injury in rats. J Neurosci 2004; 24: 3752-61.

47. Moreau T, Coles A, Wing M, Isaacs J, Hale G, Waldmann $\mathbf{H}$ et al. Transient increase in symptoms associated with cytokine release in multiple sclerosis. Brain 1996; 119: 225-37.

48. Redford EJ, Kapoor R, Smith KJ. Nitric oxide donors reversibly block axonal conduction: demyelinated axons are especially susceptible. Brain 1997; 120: $2149-57$.

49. Brinkmeier H, Aulkenmeyer P, Wollinsky KH, Rüdel R. An endogenous pentapeptide acting as sodium channel blocker in inflammatory autoimmune disorders of the central nervous system. Nat Med 2000; 6: 808-11. 